\title{
Interventional Oncology: A Snapshot of Expanding Practice
}

\begin{abstract}
Interventional oncology (IO) was a minor part of interventional radiology. However, with the development of novel technologies and introduction of innovative therapeutics with dramatic transformations in the practice of oncology, IO is facing challenges in adopting and responding to these new changes to maintain its integral role and maximize its potentials in the efficient management of patients with cancer. In this snapshot, some of the novel therapeutic approach principles relevant to IO practice will be explained briefly.
\end{abstract}

Keywords: Chemosaturation, immunoembolization, oncolytic virotherapy

\section{Introduction}

Interventional oncology (IO) has evolved from a slang term among interventional radiologists to indicate a clinically based subspecialty that represents one of the fastest growing areas in interventional radiology.

IO has been recognized as the forth pillar of cancer care through robust multidisciplinary collaboration with medical, surgical, and radiation oncology. Its growing trend continues to expand the practice due to assertive and disruptive innovations that add novel therapeutic approaches and improve the current therapeutic interventions, respectively. Moreover, oncology practice per se has recent evolutionary and revolutionary changes, so-called personalized oncology, that changed the traditional classification and management of cancer and become integral components of cancer management. ${ }^{[1]}$ Such changes and innovations lead to expanding the therapeutic choices and approaches with great efficacy, efficiency, and value and improving the lives of cancer patients.

This article will briefly explain the principles and rationale of some new therapeutic platforms that are under technical and clinical investigations and related to IO practice.

\section{Oncolytic Virotherapy}

Historically, the viruses have been used as therapeutic agents against cancer with good

This is an open access article distributed under the terms of the Creative Commons Attribution-NonCommercial-ShareAlike 3.0 License, which allows others to remix, tweak, and build upon the work non-commercially, as long as the author is credited and the new creations are licensed under the identical terms.

For reprints contact: reprints@medknow.com safety profile. Replication-defective viruses derived from adenovirus, adeno-associated virus, or retrovirus were used as vehicles for delivering exogenous genetic material to tumor cells as viral cancer gene therapy. ${ }^{[2]}$ This concept continues to have revival with significant advances in the design of oncolytic viruses with stronger oncolytic potential for effective tumor treatment.

Oncolytic virotherapy uses genetically modified viruses having oncolytic potential to abrogate pathogenicity while retaining their oncolytic activity ${ }^{[3]}$ allowing safe and biologic efficacy against specific tumors. The viruses infect cell of specific lines or with specific receptors preferentially and replicate within these tumor cells resulting in tumor destruction (viral oncolysis) by multiple mechanisms and with different kinetics in addition to evoking the immune response in the host while minimizing the risk of normal cell transformation. ${ }^{[4,5]}$ This therapeutic approach has several attractions including a very high therapeutic index, oncolytic virus amplification within tumor as it replicates, ${ }^{[6]}$ and low possibility of generating resistance and triggering of numerous cell death mechanisms..$^{[7]}$

Oncolytic virotherapy has been investigated in nearly every organ and major cancer types such as hepatocellular carcinoma, pancreatic carcinoma, and hepatic metastases from colorectal cancer with using of different virus species through various treatment routes. ${ }^{[4]}$ It has proved to be safe, ${ }^{[8]}$ but it has not yet used widely, reflecting the technical and

How to cite this article: Khankan A. Interventional oncology: A snapshot of expanding practice. Arab J Intervent Radiol 2017;1:15-9.

\section{Azzam Khankan}

Department of Medical Imaging, King Abdulaziz Medical City, Jeddah, Saudi Arabia
Address for correspondence: Dr. Azzam Khankan,

Department of Medical Imaging, King Abdulaziz Medical City, P. O. Box 9515, Jeddah 21423, Saudi Arabia.

E-mail:a.khankan@gmail.com

Access this article online

Website: www.arabjir.com

Quick Response Code:

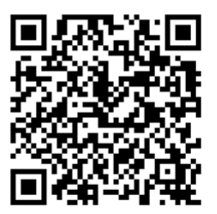


biological challenges regarding the optimal route and dose including maximum tolerated dose of virus. Systemic delivery through intravenous route is convenient and inexpensive. It is required for the treatment of multifocal or metastatic cancer, but it has some limitations such as virus sequestration in the liver and spleen, neutralization by presensitized immune system, and nonspecific binding to the vascular endothelium between the delivery site and target tumors. ${ }^{[3]}$ To overcome such limitations and ameliorate the toxicities of systemic delivery, other locoregional delivery routes have been considered to optimize the delivery of oncolytic viruses including intraarterial, intratumoral, and intracavitary routes in addition to organ isolation/perfusion. ${ }^{\left[{ }^{[9]}\right.}$ However, these routes still lead to systemic spread of amplified virus that enters the bloodstream from the lytic tumor cells, resulting in abscopal effect with infection of distance metastases. These locoregional delivery routes have been paid attention to the potential of interventional radiology techniques for facilitating such delivery using different interventional techniques under imaging guidance.

Intraarterial delivery allows selective delivery of oncolytic viruses to the target organ taking the advantage of the first-pass effect to increase concentration of viruses within the tumor. In addition, this technique allows increasing the dwell time of the virus using either embolization or balloon occlusion. Percutaneously, implantable catheter-port system can be considered for the purpose of convenience and cost-effectiveness when using therapeutic protocols of repeated doses.

Intratumoral injection has been widely used for direct administration of the oncolytic viruses. ${ }^{[10]}$ As it is difficult to control the intratumoral distribution of injectate, the infected cells will lyse and release the replicated virus which in turn infect the rest of the tumor cells which was not exposed to the viral agent at the time of injection. The injection can be performed under imaging guidance such as ultrasound, computed tomography, and magnetic resonance imaging. Using the imaging guidance may have the advantage of more selective injection into the solid tumor and avoiding the necrosis, especially when it is planned in correlation with the functional imaging.

Oncolytic virotherapy is potentially complex but promising within oncology field. However, there are ongoing researches to design more viruses with specific multifunctionality employing biotechnology-engineering methods in order to improve biodistribution, increase the tumor destruction capacity, elicit adaptive antitumor immune responses, and optimize the virotherapy.

\section{Immunoembolization}

This therapy is basically a transcatheter intraarterial therapy that used specifically in liver malignancies. It consists of infusion of biological response modifiers or immunologic agents into the hepatic artery followed by embolization. The therapeutic agents used in this approach include basal metabolic rate therapy, including cytokines, toll-like receptor agonists, ex vivo stimulated immune cells, monoclonal antibodies, and vaccines. ${ }^{[1]}$

It is based on the fact that despite the abundance of immune cells such as Kupffer macrophages, antigen-presenting cells, and innate immune cell populations in the normal liver, the liver is tolerant and its immune response is naturally suppressed to avoid overactivation of the immune system by persistent exposure to antigens and probiotics from the gastrointestinal tract. ${ }^{[12,13]}$

Different transarterial embolization techniques initially not only cause coagulation necrosis of tumors but also play a role in providing tumor antigens to the local immune system due to slight inflammatory cell infiltration in a narrow periphery on tumor necrosis. ${ }^{[14]}$ Therefore, concurrent administration of biological response modifiers will increase the dwelling time of agents and induce an inflammatory response that improves the antigen presentation to the local immune system and stimulate it. Such a local stimulation may shift the tolerogenic microenvironment of normal tissue to systemic immunostimulatory against tumor cells suppressing the growth of untreated tumors and circulating cancer cells. Thereby, immunoembolization could potentially create an in situ tumor vaccine. ${ }^{[15]}$

Previous clinical studies with using transarterial immunoembolization for different hepatic tumors have been successful with encouraging tumoricidal effects. OK-432 compound (a penicillin-killed and lyophilized preparation from Streptococcus pyogenes A3) was infused selectively into the hepatic artery followed by bland embolization with fibrinogen, thrombin, or lipiodol for the treatment of hepatocellular carcinoma. ${ }^{[16,17]}$ OK-432 stimulates macrophages and induces multiple cytokines, including interleukin (IL)-1, IL-2, interferon (IFN)-gamma, tumor necrosis factor (TNF)-alpha, IL-6, IL-8, granulocyte colony-stimulating factor (CSF), granulocyte-macrophage CSF (GM-CSF), IL-12, and IL-18. ${ }^{[18]}$ Moreover, preoperative immunoembolization with OK-432 and fibrinogen caused suppression of tumor recurrence that might be related to activation of intratumoral M1 macrophages. ${ }^{[19]}$ Pathologic examinations of surgically resected tissues treated with OK-432 showed massive infiltration of mononuclear cells, including monocytes, $\mathrm{CD}^{+} \mathrm{T}$-cells, and $\mathrm{CD}^{+} \mathrm{T}$-cells in addition to coagulation necrosis. ${ }^{[17]}$

In addition, IFN-gamma was reported in patients with hepatocellular carcinoma to be feasible and effective without significant toxicity. ${ }^{[20]}$

The approach has been pursued for the purpose of treatment of metastatic uveal melanoma using GM-CSF, an immune cells-secreted glycoprotein that increases myeloid cell production, stimulates macrophages and dendritic cells, 
and increases cytotoxicity of monocytes toward tumor cell lines through release of TNF. GM-CSF is emulsified in lipiodol and administered into the hepatic artery followed by bland embolization with Gelfoam to retain the activity after separation from the emulsion. This technique was shown to be safe and feasible in phase 1 trial for the treatment of patients with hepatic metastasis from primary uveal melanoma. ${ }^{[21]}$ When using immunoembolization with high dose of GM-CSF, the progression-free survival in extrahepatic organs and overall survival have been prolonged. ${ }^{[22]}$

Although immunoembolization is rightfully gaining more momentum as an important therapeutic strategy in the management of hepatic malignancies, more technical refinements are needed for optimizing such a promising therapy.

\section{Chemosaturation Percutaneous Hepatic Perfusion}

Traditionally, the concept of regional perfusion aims to deliver high-dose chemotherapeutics to a region of the body burdened with regional cancer using extensive surgical methods that required long hospitalization. ${ }^{[23,24]}$ Recently, newer approach using percutaneous interventional radiology techniques has been developed as an alternative regional treatment to surgical isolated hepatic perfusion for unresectable hepatic metastases from different organ malignancies. ${ }^{[25]}$

Chemosaturation percutaneous hepatic perfusion (CS-PHP) is a minimally invasive locoregional therapy that allows continuous administration of high-dose chemotherapy over the period of $1 \mathrm{~h}$ cycle time to the liver while limiting systemic toxicity by extracorporeal filtering hepatic venous blood. ${ }^{[26]}$ CS-PHP maximizes treatment to the entire liver and may result in a sustainable favorable outcome in selected patients.

The CS-PHP procedure requires well-trained multidisciplinary team consisted of interventional radiologists, anesthesiologists, and extracorporeal perfusion technologists. ${ }^{[27]}$ Currently, only one CS-PHP system is commercially available (Chemosaturation Hepatic Delivery System, Delcath Systems Inc., New York, USA), and most of the techniques are used as per the manufactures' instructions. Although the technical details of the procedure are beyond the scope of this article, it basically includes three steps:

- Isolation of hepatic perfusion by diverting the hepatic venous outflow from the circulation to an external circuit with a unique isolation-aspiration double-balloon catheter (Isofuse $^{\circledR}$ ) placed in the inferior vena cava through the femoral vein

- Saturation of the hepatic artery with high-dose chemotherapeutics during the period of time cycle through selective placed catheter after performing hepatic arterial mapping and embolizing all branches of the hepatic artery such as gastroduodenal and right gastric arteries to prevent the chemotherapeutic from leaking into the arteries that supply other organs

- Hemofiltration of the venous blood extracorporeally that is shunted out and the return in the body through the jugular vein to reduces toxicity of the chemotherapeutics in the blood before returning the blood to the body.

Different chemotherapeutics have been used for chemosaturation at different doses including doxorubicin, oxaliplatin, ${ }^{[28,29]}$ fluorouracil, ${ }^{[30]}$ paclitaxel, ${ }^{[29]}$ and melphalan. ${ }^{[29,31-35]}$ Although the efficacy of CS-PHP is likely to vary according to the type of tumor and chemotherapeutic agent, melphalan is the agent that showed both efficacy and reversible hepatic toxicity even at high doses in the treatment of unresectable isolated hepatic metastases from a variety of tumors, including melanoma, colorectal cancer, gastric cancer, hepatocellular carcinoma, neuroendocrine tumors, and sarcoma. ${ }^{[29,36,37]}$ The main adverse event of melphalan is bone marrow suppression presenting thrombocytopenia, anemia, and neutropenia. ${ }^{[38]}$ However, such toxicity is manageable. Other anecdotal adverse events include hemodynamic instability during procedure and bleeding due to unexpected disseminated intravascular coagulation.

CS-PHP is a very appealing minimally invasive regional therapy. It has particular advantages over other intraarterial therapies as the chemotherapeutic agents are filtered from the blood in addition to being alternative to surgical isolated hepatic perfusion. It is repeatable and has well-established safety profile with efficacy against a spectrum of tumor histologies. As CS-PHP concept does not prescribe a particular chemotherapeutic agent, further clinical investigations are required to determine long-term effects and standardize the technique in terms of chemotherapeutic agent choice, dose, and circulation time individually for each tumor type and specific clinical setting in addition to combination with other treatment modalities.

Moving forward, IO can expand the traditional armamentarium of therapies through translating and transition novel therapeutic concepts including molecular, biologic, immune therapies from being disruptive innovations to the standards of care. Well understanding and adopting of innovative therapeutics and technologies in addition to responding to the recent transformations in oncology practice are essential to continue and maximize the potentials of IO as a main dedicated player integrated in the modern oncology practice.

Financial support and sponsorship

Nil.

Conflicts of interest

There are no conflicts of interest. 


\section{References}

1. Abi-Jaoudeh N, Duffy AG, Greten TF, Kohn EC, Clark TW, Wood BJ. Personalized oncology in interventional radiology. J Vasc Interv Radiol 2013;24:1083-92.

2. Kuruppu D, Tanabe KK. Viral oncolysis by herpes simplex virus and other viruses. Cancer Biol Ther 2005;4:524-31.

3. Russell SJ, Peng KW, Bell JC. Oncolytic virotherapy. Nat Biotechnol 2012;30:658-70.

4. Sze DY, Reid TR, Rose SC. Oncolytic virotherapy. J Vasc Interv Radiol 2013;24:1115-22.

5. Thorne SH, Hermiston T, Kirn D. Oncolytic virotherapy: Approaches to tumor targeting and enhancing antitumor effects. Semin Oncol 2005;32:537-48.

6. Thorne SH, Tam BY, Kirn DH, Contag CH, Kuo CJ. Selective intratumoral amplification of an antiangiogenic vector by an oncolytic virus produces enhanced antivascular and anti-tumor efficacy. Mol Ther 2006;13:938-46.

7. Mahoney DJ, Stojdl DF. Molecular pathways: Multimodal cancer-killing mechanisms employed by oncolytic vesiculoviruses. Clin Cancer Res 2013;19:758-63.

8. Pandha HS. Science in focus - Oncolytic viruses: New multifunctional immunotherapeutics. Clin Oncol (R Coll Radiol) 2016;28:615-8.

9. Pencavel T, Seth R, Hayes A, Melcher A, Pandha H, Vile R, et al. Locoregional intravascular viral therapy of cancer: Precision guidance for Paris's arrow? Gene Ther 2010;17:949-60.

10. Bourke MG, Salwa S, Harrington KJ, Kucharczyk MJ, Forde PF, de Kruijf $\mathrm{M}$, et al. The emerging role of viruses in the treatment of solid tumours. Cancer Treat Rev 2011;37:618-32.

11. Yamamoto A, Sato T. Locoregional treatment of malignant hepatic tumors with biologic response modifiers. Surg Oncol Clin N Am 2008;17:935-55, xii.

12. Canning C, O’Brien M, Hegarty J, O'Farrelly C. Liver immunity and tumour surveillance. Immunol Lett 2006;107:83-8.

13. Malter M, Friedrich E, Süss R. Liver as a tumor cell killing organ: Kupffer cells and natural killers. Cancer Res 1986;46:3055-60.

14. Higuchi T, Kikuchi M, Okazaki M. Hepatocellular carcinoma after transcatheter hepatic arterial embolization. A histopathologic study of 84 resected cases. Cancer 1994;73:2259-67.

15. Sullivan K. Immunoembolization for melanoma. $1^{\text {st }}$ ed. New York: Cambridge University Press; 2008.

16. Kanai $\mathrm{T}$, Monden $\mathrm{M}$, Sakon $\mathrm{M}$, Gotoh $\mathrm{M}$, Umeshita K, Hasuike Y, et al. New development of transarterial immunoembolization (TIE) for therapy of hepatocellular carcinoma with intrahepatic metastases. Cancer Chemother Pharmacol 1994;33 Suppl 1:S48-54.

17. Yoshida T, Sakon M, Umeshita K, Kanai T, Miyamoto A, Takeda $\mathrm{T}$, et al. Appraisal of transarterial immunoembolization for hepatocellular carcinoma: A clinicopathologic study. J Clin Gastroenterol 2001;32:59-65.

18. Ryoma Y, Moriya Y, Okamoto M, Kanaya I, Saito M, Sato M. Biological effect of OK-432 (picibanil) and possible application to dendritic cell therapy. Anticancer Res 2004;24:3295-301.

19. Kenjo A, Sato T, Marubashi S, Saito T, Tsuchiya T, Kimura $\mathrm{T}$, et al. Role of intratumoral infiltrating macrophages after transarterial immunoembolization for hepatocellular carcinoma. J Hepatobiliary Pancreat Sci 2016;23:298-304.

20. Yuen MF, Ooi CG, Hui CK, Wong WM, Wong BC, Chan AO, et al. A pilot study of transcatheter arterial interferon embolization for patients with hepatocellular carcinoma. Cancer 2003;97:2776-82.

21. Sato T, Eschelman DJ, Gonsalves CF, Terai M, Chervoneva I,
McCue PA, et al. Immunoembolization of malignant liver tumors, including uveal melanoma, using granulocyte-macrophage colony-stimulating factor. J Clin Oncol 2008;26:5436-42.

22. Yamamoto A, Chervoneva I, Sullivan KL, Eschelman DJ, Gonsalves CF, Mastrangelo MJ, et al. High-dose immunoembolization: Survival benefit in patients with hepatic metastases from uveal melanoma. Radiology 2009;252:290-8.

23. Alexander HR Jr., Bartlett DL, Libutti SK. Current status of isolated hepatic perfusion with or without tumor necrosis factor for the treatment of unresectable cancers confined to liver. Oncologist 2000;5:416-24.

24. Jones A, Alexander HR Jr. Development of isolated hepatic perfusion for patients who have unresectable hepatic malignancies. Surg Oncol Clin N Am 2008;17:857-76, x.

25. Vogel A, Gupta $S$, Zeile $M$, von Haken R, Brüning $R$, Lotz $\mathrm{G}$, et al. Chemosaturation percutaneous hepatic perfusion: A systematic review. Adv Ther 2017;33:2122-38.

26. Alexander HR Jr., Butler CC. Development of isolated hepatic perfusion via the operative and percutaneous techniques for patients with isolated and unresectable liver metastases. Cancer $\mathrm{J}$ 2010;16:132-41.

27. Burgmans MC, de Leede EM, Martini CH, Kapiteijn E, Vahrmeijer AL, van Erkel AR. Percutaneous isolated hepatic perfusion for the treatment of unresectable liver malignancies. Cardiovasc Intervent Radiol 2016;39:801-14.

28. $\mathrm{Ku} \mathrm{Y}$, Iwasaki $\mathrm{T}$, Tominaga $\mathrm{M}$, Fukumoto $\mathrm{T}$, Takahashi $\mathrm{T}$, Kido $\mathrm{M}$, et al. Reductive surgery plus percutaneous isolated hepatic perfusion for multiple advanced hepatocellular carcinoma. Ann Surg 2004;239:53-60.

29. Uzgare RP, Sheets TP, Johnston DS. Evaluation of melphalan, oxaliplatin, and paclitaxel in colon, liver, and gastric cancer cell lines in a short-term exposure model of chemosaturation therapy by percutaneous hepatic perfusion. Anticancer Res 2013;33:1989-2000.

30. Ravikumar TS, Pizzorno G, Bodden W, Marsh J, Strair R, Pollack J, et al. Percutaneous hepatic vein isolation and high-dose hepatic arterial infusion chemotherapy for unresectable liver tumors. J Clin Oncol 1994;12:2723-36.

31. Marinelli A, Vahrmeijer AL, van de Velde CJ. Phase I/II studies of isolated hepatic perfusion with mitomycin $\mathrm{C}$ or melphalan in patients with colorectal cancer hepatic metastases. Recent Results Cancer Res 1998;147:83-94.

32. Vahrmeijer AL, van Dierendonck JH, Keizer HJ, Beijnen JH, Tollenaar RA, Pijl ME, et al. Increased local cytostatic drug exposure by isolated hepatic perfusion: A phase I clinical and pharmacologic evaluation of treatment with high dose melphalan in patients with colorectal cancer confined to the liver. Br $\mathrm{J}$ Cancer 2000;82:1539-46.

33. de Vries MR, Borel Rinkes IH, van de Velde CJ, Wiggers T, Tollenaar RA, Kuppen PJ, et al. Isolated hepatic perfusion with tumor necrosis factor alpha and melphalan: Experimental studies in pigs and phase I data from humans. Recent Results Cancer Res 1998;147:107-19.

34. van Iersel LB, de Leede EM, Vahrmeijer AL, Tijl FG, den Hartigh J, Kuppen PJ, et al. Isolated hepatic perfusion with oxaliplatin combined with $100 \mathrm{mg}$ melphalan in patients with metastases confined to the liver: A phase I study. Eur J Surg Oncol 2014;40:1557-63.

35. van Iersel $\mathrm{LB}$, Gelderblom $\mathrm{H}$, Vahrmeijer $\mathrm{AL}$, van Persijn van Meerten EL, Tijl FG, Putter $\mathrm{H}$, et al. Isolated hepatic melphalan perfusion of colorectal liver metastases: Outcome and prognostic factors in 154 patients. Ann Oncol 2008;19:1127-34.

36. Pingpank JF, Libutti SK, Chang R, Wood BJ, Neeman Z, 
Kam AW, et al. Phase I study of hepatic arterial melphalan infusion and hepatic venous hemofiltration using percutaneously placed catheters in patients with unresectable hepatic malignancies. J Clin Oncol 2005;23:3465-74.

37. Deneve JL, Choi J, Gonzalez RJ, Conley AP, Stewart S, Han D, et al. Chemosaturation with percutaneous hepatic perfusion for unresectable isolated hepatic metastases from sarcoma. Cardiovasc Intervent Radiol 2012;35:1480-7.

38. Lillemoe HA, Alexander HR. Current status of percutaneous hepatic perfusion as regional treatment for patients with unresectable hepatic metastases: A review. Am Oncol Hematol Rev 2014;15-23. 